\title{
Chronic Neutrophilic Leukemia with Monocytosis
}

\author{
Laila Alromaih $^{1}$, Leena Abdalla ${ }^{1}$, Arifa Jamal ${ }^{1}$, Assim Osman $^{1}$, Mohanad Bakkar $^{1}$, Lina Abdul Samad ${ }^{2}$, Tamer M Ahmed $^{3}$ \\ ${ }^{1}$ Internal Medicine Department, KFMMC, Dhahran, Kingdom of Saudi Arabia \\ ${ }^{2}$ Radiology Department, Nuclear medicine division, KFMMC Dhahran, Kingdom of Saudi Arabia \\ ${ }^{3}$ Clinical Hematology and Internal Medicine, Ain Shams University, Cairo, Egypt
}

How to cite this article: Alromaih L, Abdalla L, Jamal A ,Osman A, Bakkar M, Samad LA ,Ahmed TM. Chronic neutrophilic leukemia with monocytosis. EJCRIM 2021;8: doi:10.12890/2021_002595.

Conflicts of Interests: The authors declare there are no competing interests.

This article is licensed under a Commons Attribution Non-Commercial 4.0 License

\section{ABSTRACT}

Chronic neutrophilic leukaemia is a very rare disease with diagnosis based on persistent leucocytosis $>25 \times 10^{3} / \mu \mathrm{l}$ and $\mathrm{monocytes}<1 \times 10^{3} / \mu$ l. The revised WHO criteria 2016 included CSF3R gene mutations as a diagnostic finding.

We report the case of a 77-year-old man who was found to have asymptomatic persistent mature neutrophilic leucocytosis with monocytosis. Molecular study confirmed the presence of a CSF3R gene mutation in the absence of morphological or genetic features of myelodysplasia or other forms of myelodysplastic syndrome.

The patient's medical history was significant for coronary artery disease, hypertension, chronic obstructive pulmonary disease, bilateral cystic bronchiectasis, moderate pulmonary hypertension, tuberculosis treated 27 years previously, hypothyroidism, and a thyroid nodule. He had hepatosplenomegaly but no lymphadenopathy, and no other malignancy was seen on computed tomography (CT) scanning. At the time of evaluation, he was free of symptoms and had no evidence of infection or drug-induced leucocytosis.

The patient was referred to an oncology centre and treated with hydroxyurea and subsequently azacitidine. However, he developed pancytopenia with bone marrow aplasia. He died with neutropenia sepsis.

The presence of persistent monocytosis in this case created a diagnostic dilemma as to whether the disease was a variant of chronic neutrophilic leukaemia or was reactive monocytosis.

\section{LEARNING POINTS}

- The presence of a CSF3R gene mutation is diagnostic for chronic neutrophilic leukaemia (CNL).

- The monocytosis in this patient might have been a new variant of CNL.

\section{KEYWORDS}

Chronic neutrophilic leukaemia, myeloproliferative neoplasm, colony-stimulating factor 3 receptor mutation, atypical chronic myeloid leukaemia, chronic myelomonocytic leukaemia

\section{INTRODUCTION}

Chronic neutrophilic leukaemia (CNL) is a BCR-ABL1 negative myeloproliferative neoplasm (MPN). CNL is defined by the presence of predominantly mature neutrophilic proliferation, bone marrow granulocytic hyperplasia, and hepatosplenomegaly. Colony-stimulating factor 3 receptor (CSF3R) mutations were added to the 2016 WHO CNL diagnostic criteria ${ }^{[1]}$. We describe a case of CSF3R-mutated CNL with monocytosis, which might have been a variant of the CNL presentation. 


\section{CASE DESCRIPTION}

A 77-year-old male heavy smoker was recalled to hospital due to a high white cell count of $35 \times 10^{3} / \mathrm{ml}$ found during routine laboratory testing for a regular outpatient appointment in King Fahd Military Medical Complex, Dhahran. The patient was admitted to hospital with borderline hypotension, but no clear focus of infection was found.

There was no history of fever, skin rash or bleeding tendency. There were no symptoms related to other systems.

The patient's medical history was significant for coronary artery disease, hypertension, chronic obstructive pulmonary disease, bilateral cystic bronchiectasis, moderate pulmonary hypertension, tuberculosis (TB) treated 27 years previously, hypothyroidism, a colloid/ adenomatous thyroid nodule, and iron-deficiency anaemia.

Physical examination revealed moderate hepatomegaly and massive splenomegaly. No lymphadenopathy or petechial haemorrhages were seen.

Initial blood work-up showed a white blood cell count of $35 \times 10^{3} / \mu$, with neutrophil count $24.2 \times 10^{3} / \mu$ l, basophil count $0.112 \times 10^{3} / \mu$, eosinophil count $0.223 \times 10^{3} / \mu \mathrm{l}$, monocyte count $6.3 \times 10^{3} / \mu \mathrm{l}$, and lymphocytes $1.5 \times 10^{3} / \mu \mathrm{l}$. Haemoglobin was $11.2 \mathrm{~g} / \mathrm{dl}(12-16), \mathrm{MCV} 81.5 \mathrm{fl}$, $\mathrm{MCH} 26.5 \mathrm{pg}$, haematocrit $34.5 \%$ and platelet count $266 \times 10^{3} / \mu \mathrm{l}$ (140-450). Leukocyte alkaline phosphatase was 158 (10-100).

The patient underwent full work-up for possible underlying infection and was started on empirical intravenous antibiotics. However, his inflammatory markers, sputum, blood and urine cultures and the chest x-ray were all unremarkable. Repeated cultures including AFB culture and smear were all negative.

Chest and abdominal computed tomography (CT) scans were performed and showed bilateral basal lung infiltrations with a tree-in-bud pattern but no evidence of active TB, and splenic enlargement.

Review of the patient's medical file revealed persistent leucocytosis of $25-35 \times 10^{3} / \mu$ l with predominant neutrophilia present for over a year. The patient was not on oral steroids during or prior to hospital admission, and inhaled steroids were held so true neutrophilic leucocytosis could be evaluated.

A differential diagnosis of CNL versus chronic myelomonocytic leukaemia was suggested. A peripheral blood film (Fig. 1) was consistent with neutrophilic leucocytosis and monocytosis.

A bone marrow sample showed hyperplastic aspirate (Fig. 2) with reactive marrow with trilineage haematopoiesis, myeloid hyperplasia with normal maturation and polymorphonuclear cells and macrophages. No dysplasia was identified.

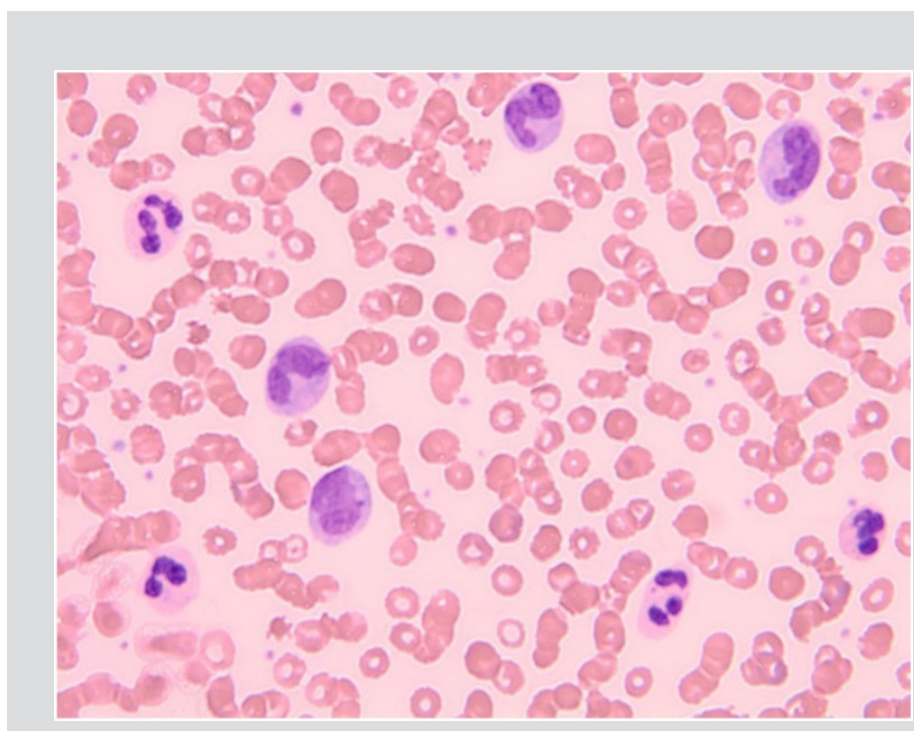

Figure 1. Peripheral blood film showing leucocytosis with neutrophilia and monocytosis (high power)

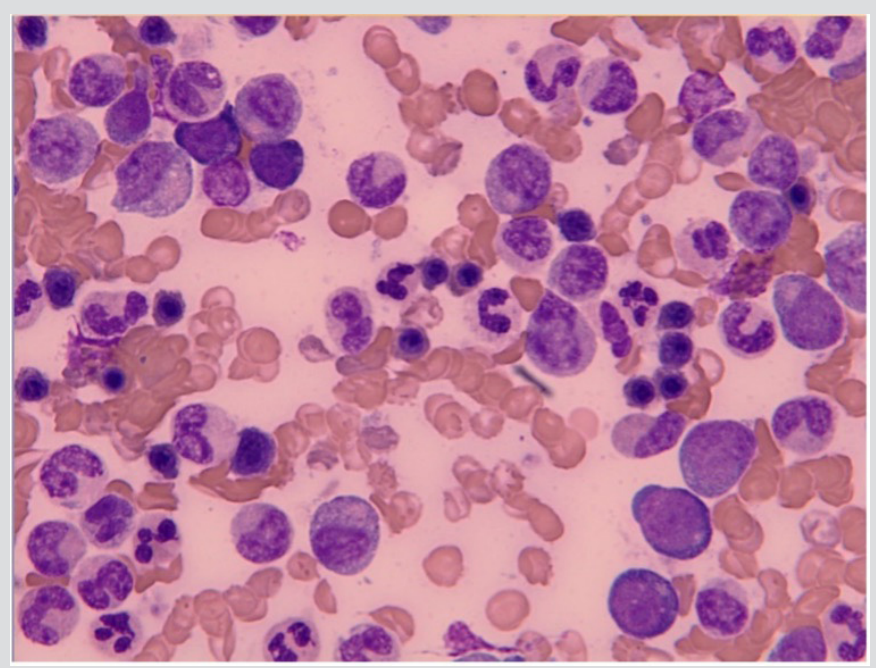

Figure 2. Bone marrow sample showing chronic granulocytic leukaemia (high power)

Cytogenetic analysis of the bone marrow sample indicated the existence of a pathological cell line with a translocation between the long arm of chromosome 1 and the long arm of chromosome 5 ( $t(1 ; 5)(q 12 ; q 12))$. Of note, the breakpoints described in the literature are different from the breakpoints found in our patient. 
Molecular genetic analysis was carried out and detected a mutation in the CSF3R gene (c.1853C>T) with a mutational burden of 31\%. Analysis of leukaemia-related fusion genes BCR-ABL1, CALM-AF10, CBFB-MYH11, DEK-NUP214 (DEK-CAN), KMT2A-AFDN (MLL-AF6), KMT2A-MLLT3 (MLL-AF9), KMT2A-ELL (MLL-ELL), KMT2A-PTD (MLL-PTD), NPM1-MLF1, PML-RARA and RUNX1-RUNX1T1 (AML1ETO) did not detect any mutations. Analysis of known genes for myelodysplastic syndrome (MDS) was carried out, and mutations in genes ASXL1, KRAS, SETBP1, U2AF1 and ZRSR2 were also detected with a mutational burden of each gene of 37\%, 41\%, 46\%, 42\%, and 84\%, respectively. The remaining known gene mutations in BCOR, CBL, DNMT3A, ETV6, EZH2, FLT3, GATA2, IDH1, IDH2, JAK2, MPL, NPM1, NRAS, PTPN11, RAD21, RUNX1, SF3B1, SRSF2, STAG2, TET2, TP53 and WT1 were not detected.

A gallium scan (Fig. 3) showed interrupted bone marrow uptake over the mid-shaft of the right femur which was less likely to be a normal variant. A follow-up MRI of the right femur revealed enhanced abnormal red marrow reconversion, which could indicate infiltrative bone marrow disease. To complete the work-up, an x-ray was carried out and showed coarsening of the bony trabecula at the mid-femoral shaft with no definite cortical destruction, no destructive bony lesions, and no definite periosteal reaction.

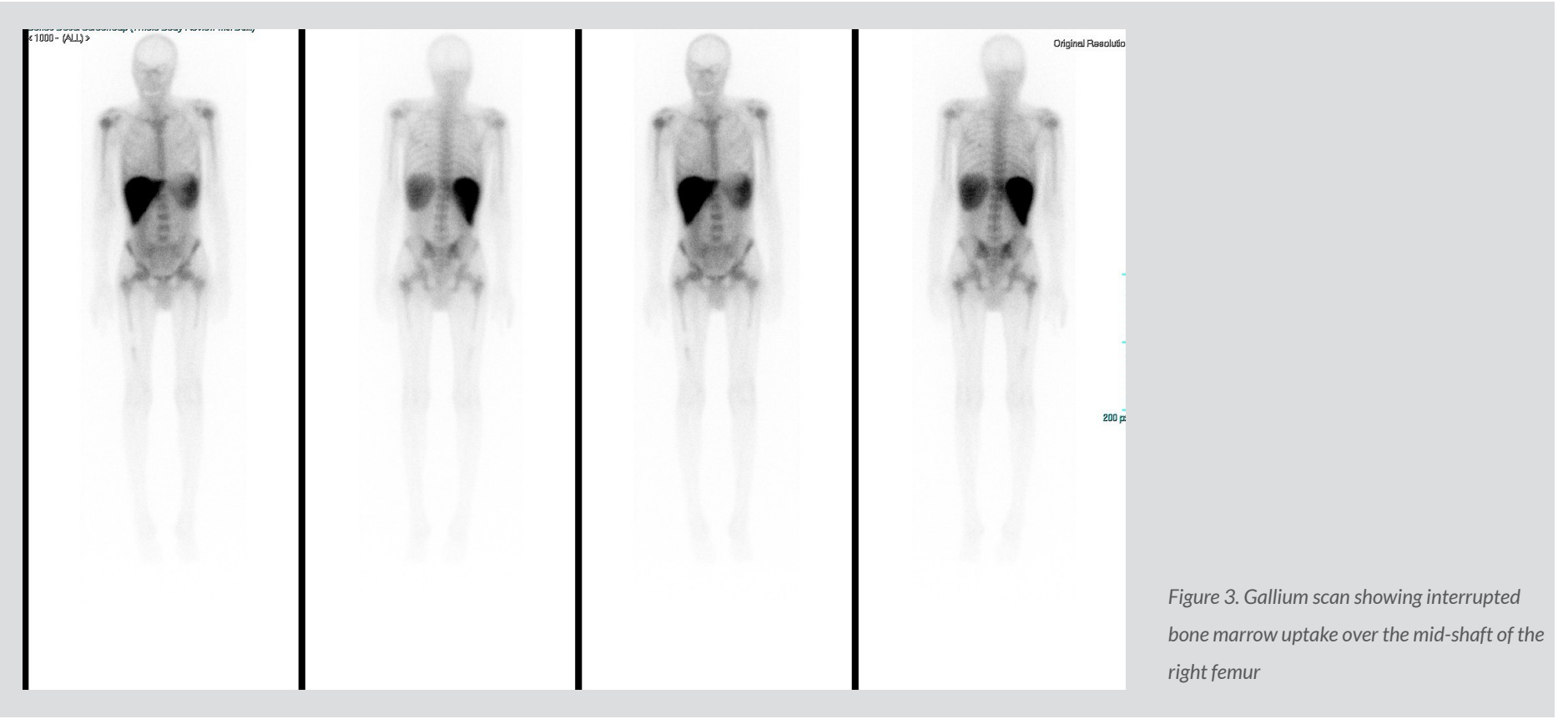

The patient was referred to an oncology centre with the diagnosis of CSF3R-mutated CNL and was started on hydroxyurea. We followed him for 6 months. During the first few months, his WBC count started to improve, but later he developed thrombocytopenia and then pancytopenia and became dependent on blood product transfusions. Azacitidine was initiated as there was no improvement in his condition. Unfortunately, he died with febrile neutropenia, and hypocellular/aplastic marrow and erythroid precursors-normoblast were seen on a repeat bone marrow biopsy.

\section{DISCUSSION}

CNL is a rare BCR-ABL1 negative MPN. The most common presenting symptom is fatigue, as described in the literature, but most patients present asymptomatically with incidental leucocytosis found on blood work-up ${ }^{[2]}$. The WHO 2016 revised diagnostic criteria for CNL included the CSF3RT618I gene mutation to aid diagnosis and molecularly targeted therapy (Table 1). Other diagnostic criteria include leucocytosis of $\geq 25 \times 10^{9} /$ with $\geq 80 \%$ neutrophils, with $<10 \%$ circulating neutrophil precursors with blasts rarely observed, hypercellular bone marrow, and a negative myelodysplastic syndrome (MDS) gene panel ${ }^{[1]}$.

It is crucial to discuss other causes of mature peripheral neutrophilia, such as atypical chronic myeloid leukaemia (aCML) and chronic myelomonocytic leukaemia (CMML).

Atypical CML is also BCR-ABL1 negative in the WHO 2016 revised criteria, which emphasized the presence of molecular changes (ETNK1, SETBP1), with persistent leucocytosis $\geq 13 \times 10^{9} /$ w with immature circulating myeloid precursors with $\geq 10 \%$ leukocytes, an absence of basophilia and monocytosis, and hypercellular bone marrow with granulocytic proliferation and granulocytic dysplasia ${ }^{[3]}$ (Table 1). 


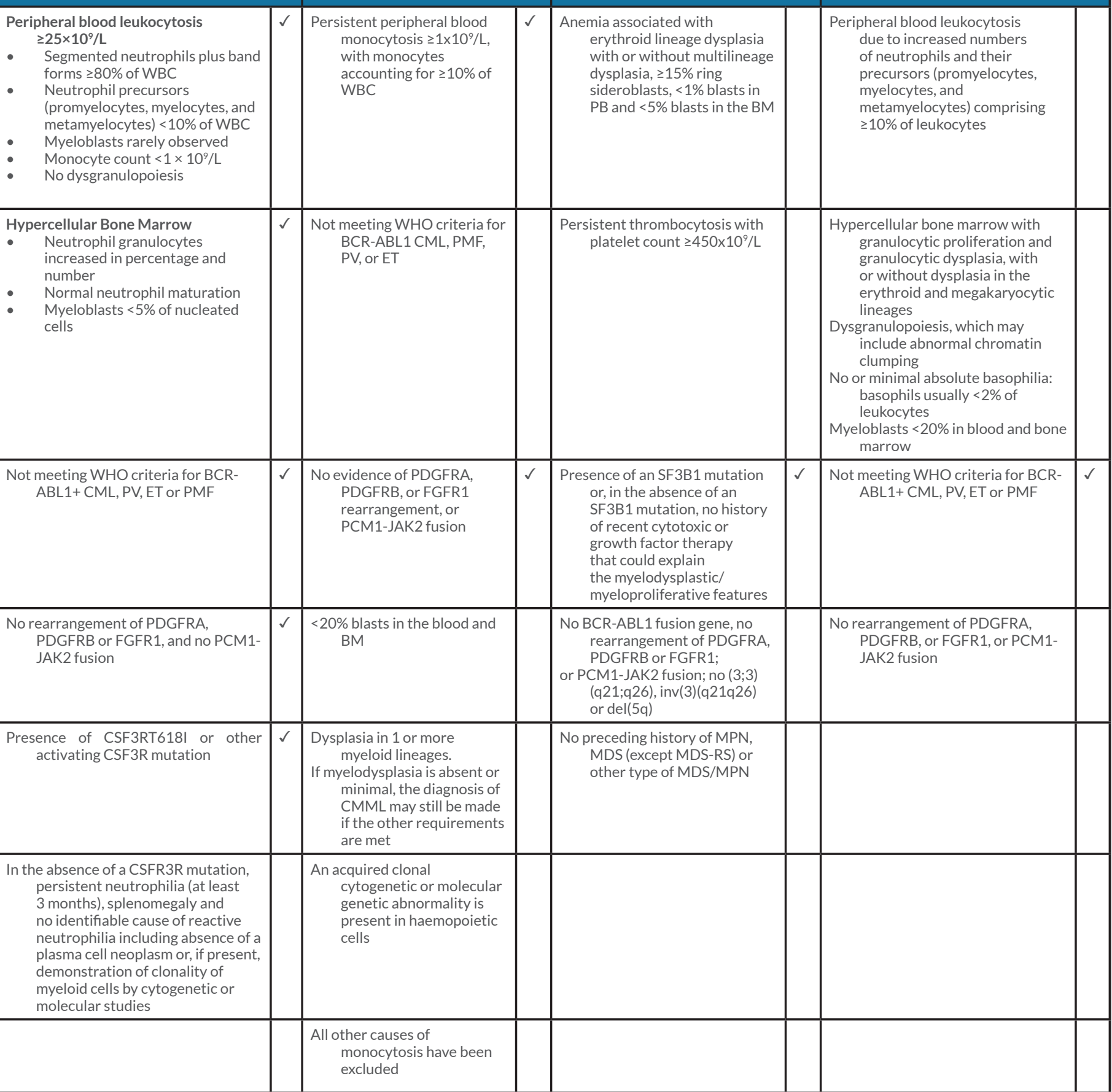

Table 1 The WHO 2016 revised criteria for chronic neutrophilic leukaemia, chronic myelomonocytic leukaemia, myelodysplastic syndrome/myeloproliferative neoplasm and atypical chronic myeloid leukaemia. Ticks indicate the criteria that fit our case aCML, atypical chronic myeloid leukaemia; BM, bone marrow; CMML, chronic myelomonocytic leukaemia; CNL, chronic neutrophilic leukaemia; ET, essential thrombocythaemia; MDS, myelodysplastic syndrome; MDS/MPN, myelodysplastic syndrome/myeloproliferative neoplasm; MDS-RS, myelodysplastic syndromes with ring sideroblasts; MPN, myeloproliferative neoplasm; PB, peripheral blood; PMF, primary myelofibrosis; PV, polycythaemia vera. 
CMML is a clinically heterogeneous disorder that is currently classified by WHO as overlapping MDS/myeloproliferative neoplasm (MPN) ${ }^{[4]}$. Diagnosis requires the presence of both absolute monocytosis $\geq 1 \times 10^{9} /$ and relative monocytosis $\geq 10 \%$ in peripheral blood, as per the WHO 2016 revised criteria. The presence of MPN features in the bone marrow and/or of MPN-associated mutations such as JAK2, CALR and MPL, tend to support a diagnosis of MPN with monocytosis rather than CMML.

MDS are a group of clonal haematopoietic disorders characterized by ineffective haematopoiesis, haematopoietic cell dysplasia, bone marrow failure, and peripheral pancytopenias ${ }^{[5]}$.

Concurrent mutations in ASXL1 and SETBP1 in CSF3R-mutated CNL patients were some of the mutations described in literature. The presence of ASXL1 was independently predictive of reduced survival and the presence of ASXL1 and lack of SETBP1 were indicative of CNL to CMML evolution ${ }^{[5]}$.

The concomitant presence of monocytosis in our patient could have been a variant of CNL presentation, or due to the multiple comorbidities and previous TB infection, even though there was no evidence of TB activation or active infection.

In conclusion, CNL is very rare and the literature on this disease is lacking. CNL has an aggressive course and must be distinguished from other conditions including reactive leucocytosis, leukemoid reaction, aCML and CMML. The presence of a specific mutation (CSF3R) can help in confirming the diagnosis of CNL and with disease management. Further studies are needed to clarify whether monocytosis in CNL is a variant presentation or a contributing factor.

\section{REFERENCES}

Szuber N, Elliot M, Tefferi A. Chronic neutrophilic leukemia: 2020 update on diagnosis, molecular genetics, prognosis, and management. Am J Hematol 2019;95(2):212-224. Elliott MA, Tefferi A. Chronic neutrophilic leukemia: 2018 update on diagnosis, molecular genetics and management. Am J Hematol 2018;93(4):578-587.

Arber DA, Orazi A, Hasserjian R, Thiele J, Borowitz MJ, Le Beau MM, et al. The 2016 revision to the World Health Organization classification of myeloid neoplasms and acute leukemia. Blood 2016;127(20):2391-2405.

4. Patnaik MM, Tefferi A. Chronic myelomonocytic leukemia: 2018 update on diagnosis, risk stratification and management. Am J Hematol 2018;93(6):824-840.

5. Hong M, He G. The 2016 Revision to the World Health Organization classification of myelodysplastic syndromes. J Transl Int Med 2017;5(3):139-143. 